\title{
Design of broadband and high-output power uni-traveling-carrier photodiodes
}

\author{
Rong Zhang, ${ }^{1,2}$ Bouchaib Hraimel, ${ }^{1}$ Xue Li ${ }^{3}$ Peng Zhang, ${ }^{1}$ and Xiupu Zhang ${ }^{1, *}$ \\ ${ }^{I}$ Advanced Photonic Systems Lab., Department of Electrical and Computer Engineering, Concordia University, \\ Montreal, Quebec H3G1M8, Canada \\ ${ }^{2}$ Key Laboratory of Terahertz Solid-State Technology, Shanghai Institute of Microsystem and Information \\ Technology, Chinese Academy of Sciences, Shanghai, 200050, China \\ ${ }^{3}$ Shanghai Institute of Technical Physics, Chinese Academy of Sciences, Shanghai, 200083, China \\ xzhang@ece.concordia.ca
}

\begin{abstract}
In this paper, physically-based simulations are carried out to investigate and design broadband and high-output power uni-traveling carrier (UTC) photodiodes. The physical model is first verified by comparison to experimentally measured results. The graded-bandgap structure, which can induce potential gradient, is considered to be used in the absorption layers. It is shown that the electric field in the absorption layer is increased by the gradient, thus the performance of bandwidth and saturation current is improved by $36.6 \%$ and $40 \%$ respectively for our considered photodiode. Moreover, a modified graded-bandgap structure is proposed to further increase the electric field, and an additional $9.5 \%$ improvement in bandwidth is achieved. The final proposed UTC-PD structures will result in $399-\mathrm{GHz}$ bandwidth and $49-\mathrm{mA} \mathrm{DC}$ saturation current.
\end{abstract}

C2013 Optical Society of America

OCIS codes: (230.5170) Photodiodes; (250.0040) Detectors; (060.2330) Fiber optics communications.

\section{References and links}

1. C. Cox, E. Ackerman, G. Betts, and J. Prince, "Limits on the performance of RF-over-fiber links and their impact on device design," IEEE Trans. Microw. Theory Tech. 54(2), 906-920 (2006).

2. J.-W. Shi, C.-B. Huang, and C.-L. Pan, "Millimeter-wave photonic wireless links for very high data rate communication," NPG Asia Mater. 3(4), 41-48 (2011).

3. M. Achouche, G. Glastre, C. Caillaud, M. Lahrichi, M. Chtioui, and D. Carpentier, "InGaAs communication photodiodes: from low- to high-power-level designs," IEEE Photonics J. 2(3), 460-468 (2010).

4. H. Ito, S. Kodama, Y. Muramoto, T. Furuta, T. Nagatsuma, and T. Ishibashi, "High-speed and high-output InPInGaAs unitraveling-carrier photodiodes,” IEEE J. Sel. Top. Quantum Electron. 10(4), 709-727 (2004).

5. T. Ishibashi, N. Shimizu, S. Kodama, H. Ito, T. Nagatsuma, and T. Furuta, "Uni-traveling-carrier photodiodes," in Ultrafast Electronics Optoelectronics OSA Spring Topical Meeting, Technical Digest (Optical Society of America, 1997), pp. 166-168.

6. T. Ishibashi, S. Kodoma, N. Shimizu, and T. Furuta, "High-speed response of uni-traveling-carrier photodiodes," Jpn. J. Appl. Phys. 36(Part 1, No. 10), 6263-6268 (1997).

7. M. Chtioui, A. Enard, D. Carpentier, S. Bernard, B. Rousseau, F. Lelarge, F. Pommereau, and M. Achouche, "High-performance uni-traveling-carrier photodiodes with a new collector design," IEEE Photon. Technol. Lett. 20(13), 1163-1165 (2008).

8. D.-H. Jun, J.-H. Jang, I. Adesida, and J.-I. Song, "Improved efficiency bandwidth product of modified unitraveling carrier photodiode structures using an undoped photo-absorption layer," Jpn. J. Appl. Phys. 45(4B), 3475-3478 (2006).

9. X. Wang, N. Duan, H. Chen, and J. C. Campbell, "InGaAs-InP photodiodes with high responsivity and high saturation power," IEEE Photon. Technol. Lett. 19(16), 1272-1274 (2007).

10. M. Chtioui, F. Lelarge, A. Enard, F. Pommereau, D. Carpentier, A. Marceaux, F. van Dijk, and M. Achouche, "High responsivity and high power UTC and MUTC GaInAs-InP photodiodes," IEEE Photon. Technol. Lett. 24(4), 318-320 (2012).

11. Z. Li, H. Pan, H. Chen, A. Beling, and J. C. Campbell, "High-saturation-current modified uni-traveling-carrier photodiode with cliff layer,” IEEE J. Quantum Electron. 46(5), 626-632 (2010).

12. J.-W. Shi, F.-M. Kuo, and J. E. Bowers, "Design and analysis of ultra-high-speed near-ballistic uni-travelingcarrier photodiodes under a $50-\Omega$ load for high-power performance," IEEE Photon. Technol. Lett. 24(7), 533$535(2012)$. 
13. J.-W. Shi, Y.-S. Wu, C.-Y. Wu, P.-H. Chiu, and C.-C. Hong, "High-speed, high-responsivity, and high-power performance of near-ballistic uni-traveling-carrier photodiode at $1.55-\mu \mathrm{m}$ wavelength," IEEE Photon. Technol. Lett. 17(9), 1929-1931 (2005).

14. M. Chtioui, A. Enard, D. Carpentier, S. Bernard, B. Rousseau, F. Lelarge, F. Pommereau, and M. Achouche, "High-power and high-linearity uni-traveling-carrier photodiodes for analog photonic links," IEEE Photon. Technol. Lett. 20(3), 202-204 (2008).

15. H. Fukano, Y. Muramoto, K. Takahata, and Y. Matsuoka, "High efficiency edge-illuminated unitravellingcarrier-structure refracting-facet photodiode," Electron. Lett. 35(19), 1664-1665 (1999).

16. S. Demiguel, N. Li, X. Li, X. Zheng, J. Kim, J. C. Campbell, H. Lu, and A. Anselm, "Very high-responsivity evanescently coupled photodiodes integrating a short planar multimode waveguide for high-speed applications," IEEE Photon. Technol. Lett. 15(12), 1761-1763 (2003).

17. J. Klamkin, S. M. Madison, D. C. Oakley, A. Napoleone, F. J. O’Donnell, M. Sheehan, L. J. Missaggia, J. M. Caissie, J. J. Plant, and P. W. Juodawlkis, "Uni-traveling-carrier variable confinement waveguide photodiodes," Opt. Express 19(11), 10199-10205 (2011).

18. M. Hosseinifar, V. Ahmadi, and G. Abaeiani, "Microring-based unitraveling carrier photodiodes for high bandwidth-efficiency product photodetection in optical communication," J. Lightwave Technol. 29(9), 1285$1292(2011)$.

19. J.-W. Shi, F.-M. Kuo, C.-J. Wu, C. L. Chang, C.-Y. Liu, C. Y. Chen, and J.-I. Chyi, "Extremely high saturation current-bandwidth product performance of a near-ballistic uni-traveling-carrier photodiode with a flip-chip bonding structure,” IEEE J. Quantum Electron. 46(1), 80-86 (2010).

20. Y.-S. Wu, J.-W. Shi, and P.-H. Chiu, "Analytical modeling of a high-performance near-ballistic uni-travelingcarrier photodiode at a 1.55- $\mu \mathrm{m}$ wavelength," IEEE Photon. Technol. Lett. 18(8), 938-940 (2006).

21. R. Stratton, "Diffusion of hot and cold electrons in semiconductor barriers," Phys. Rev. 126(6), 2002-2014 (1962).

22. R. Stratton, "Semiconductor current-flow equations (diffusion and degeneracy)," IEEE Trans. Electron. Dev. 19(12), 1288-1292 (1972).

23. New semiconductor materials, characteristics and properties, http://www.ioffe.rssi.ru/SVA/NSM/.

24. S. Adachi, Physical Properties of III-V Semiconductor Compounds InP, InAs, GaAs, GaP, InGaAs, and InGaAsP, (John Wiley and Sons, 1992).

\section{Introduction}

Broadband and high-output-power photodiodes (PDs) are considered the key optical-toelectrical signal interface devices for future high-frequency and high-power fiber-optic systems, such as millimeter-wave photonic gigabit communication systems and high-speed coherent optical communication systems [1-3]. For conventional PDs, the speed performance is substantially limited by the inherent trade-off between the saturation current (related to the output power) and the resistance-capacitance (RC) limited bandwidth [2-4]. To overcome this problem, the uni-traveling-carrier PD (UTC-PD) was proposed [3-6]. Because only electrons are the active carriers in the UTC-PD, the slow hole transport is eliminated and the bandwidth and saturation-current performance is improved. In the past decade, the performance of UTC-PDs has been significantly improved by novel structure designs [7-14]. A non-uniform doped collector was designed to relax the space charge effect [7]. The modified UTC-PD (MUTC-PD) with partially depleted absorption layer was proposed to improve the bandwidth and the quantum efficiency [8-10]. Further increase of the saturation current of the MUTC-PD has been achieved by incorporating a cliff layer before the collector [11]. Near-ballistic UTC-PD (NBUTC-PD) has been demonstrated to show superior output power performance $[12,13]$. In the NBUTC-PD, a thin p-type charge layer is inserted in the collector layer to control the internal distribution of electric field, which makes the device operate under higher optimum bias voltage. On the other hand, various optical coupling techniques have also been developed. For instance, edge-illuminated UTC-PD [15], waveguide-based evanescently coupled UTC-PD [16, 17], and micro-ring-based UTC-PD [18] were all demonstrated to show improved performance. In addition, flip-chip bonding has been implemented to minimize the device heating problem, which greatly improves the highpower performance [19]. The recently reported UTC-PDs exhibit bandwidth and saturation current of $180 \mathrm{GHz}$ and $37 \mathrm{~mA}$ [19], $24 \mathrm{GHz}$ and $144 \mathrm{~mA}$ [11], and $250 \mathrm{GHz}$ and $17 \mathrm{~mA}$ [12].

To simulate the device performance, equivalent-circuit models were developed including carrier-transit-time and the RC-delay-time bandwidth-limiting factors. These parameters can be extracted by fitting the values of the circuit components to match with the experimental 
results $[12,20]$. Therefore, this method relies on measurement of the priory-fabricated device. As an alternative, physically-based modeling of UTC-PDs will be presented in this study. The accuracy of the modeling will be verified by simulating the UTC-PDs presented in [11, 12], and then a structure design with graded bandgap absorption layer will be investigated. Based on the modeling results, a novel modified graded bandgap structure by splitting the absorption layer will be proposed to improve the bandwidth performance. It will be shown that the physically-based modeling can be used to accurately predict the intrinsic behavior of UTCPDs and to directly design the device structure, and our proposed UTC-PDs with the modified graded bandgap absorption layer will improve the bandwidth and saturation current effectively.

\section{Physical modeling}

\subsection{Simulator}

A commercial physically-based simulator is used in our simulations. The physical modeling employs a comprehensive set of physical models including drift-diffusion transport model, hot carrier transport model, concentration, electric field and temperature dependent mobility models, carrier generation and recombination models, etc, and powerful numerical techniques, such as Gummel, Newton, and block-Newton nonlinear iteration strategies. As the key of our study, the hot carrier transport model is enabled in our simulation. This model follows the derivation by Stratton [21,22], which is derived starting from the Boltzmann Transport Equation. Compared to drift-diffusion model, it introduces new independent variables for electron and hole temperatures, adds continuity equations for the carrier temperatures, and treats mobility also as functions of the carrier temperatures. Therefore, nonlocal transport effects such as velocity overshoot and diffusion associated with the carrier temperature, which are neglected in conventional drift-diffusion model but are considered important to simulate submicron devices, can be included in this model. To simulate a device, the following steps are carried out: (1) Define the device structure. (2) Mesh the structure with a fine grid $(1 \mathrm{~nm})$ to ensure the accuracy. (3) Set the material properties. Most parameters for commonly used semiconductors, such as InP and InGaAs, are preset in the simulator, and other parameters are found on the website [23]. (4) Define the optical source. (5) Set variables such as bias, light power, frequency, etc. After these steps, a simulation can be run with the physical models and numerical methods mentioned above. Alternating current (AC) simulation is completed with an ideal $50 \Omega$ load. The bandwidth of the device is evaluated from the frequency response to optical sources. The photocurrent $(I)$ versus frequency $(f)$ is calculated by sweeping the frequency from DC to $500 \mathrm{GHz}$. Since the radio frequency (RF) power is related to $I^{2}$, the $-3-\mathrm{dB}$ RF response bandwidth $f_{-3 \mathrm{~dB}}$ is obtained from $10 \lg \left[I^{2}\left(f_{-3 \mathrm{~dB}}\right) / I_{\mathrm{DC}}^{2}\right]=-3$.

\subsection{Simulation of UTC-PDs}

To verify the physical modeling, we simulate the MUTC1 reported in [11] and NBUTC-PD reported in [12], labeled here as device PA and PB, respectively. PA has a $1.05-\mu \mathrm{m}$ partiallydepleted absorption layer $(0.9-\mu \mathrm{m}$ undepleted followed by $0.15-\mu \mathrm{m}$ depleted) and a $0.9-\mu \mathrm{m}$ collector. By inserting a cliff layer before the collector, PA exhibits a high-saturation-current performance $(144 \mathrm{~mA})$. PB has a relative thin absorption layer and collector, which is designed for high-frequency operation $(250 \mathrm{GHz})$. The detailed layer parameters are given in Tables 1 and 2 for PA and PB, respectively. For PA, Fig. 1(a) shows simulated modulation response for four active area diameters of $28,34,40$, and $56 \mu \mathrm{m}$, and for comparison measured bandwidths are also indicated [11]. A series resistance of $15 \Omega$ is included in the simulation for PA to fit the experimental results. The reported measured bandwidths of PA are $23 \mathrm{GHz}, 20 \mathrm{GHz}, 15 \mathrm{GHz}$, and $8 \mathrm{GHz}$ at device diameters of $28,34,40$, and $56 \mu \mathrm{m}$, respectively. Correspondingly, our simulated bandwidths are $24.3 \mathrm{GHz}, 20.5 \mathrm{GHz}, 15.9 \mathrm{GHz}$, and $9.7 \mathrm{GHz}$ as shown in Fig. 1(a). For PB, Fig. 1(b) shows the simulated modulation 
response at two bias voltages of -2 and $-5 \mathrm{~V}$, and also the measured modulation response at $-2 \mathrm{~V}$ is also shown for comparison. It is seen that a good agreement between the simulation and measurement is achieved. The experimental results in [12] showed that there is a slight degradation of bandwidth at higher bias of $-5 \mathrm{~V}$, which is also predicted as shown in Fig. 1(b) inset.

Table 1. Layer Parameters of PA in [11] and PA with Graded-bandgap Absorption Layer

\begin{tabular}{|c|c|c|c|c|}
\hline Layer & $\mathrm{PA}$ in [11] & PA with graded bandgap & Thickness $(\AA)$ & $\begin{array}{l}\text { Doping }\left(\mathrm{cm}^{-3}\right) \\
\text { /Type }\end{array}$ \\
\hline P-contact & & $\mathrm{In}_{0.53} \mathrm{Ga}_{0.47} \mathrm{As}$ & 500 & $2 \times 10^{19} / \mathrm{P}$ \\
\hline Block & & $\operatorname{InP}$ & 1000 & $1 \times 10^{18} / \mathrm{P}$ \\
\hline \multirow[t]{2}{*}{ Transition } & & InGaAsP & 300 & $2 \times 10^{18} / \mathrm{P}$ \\
\hline & & $\mathrm{In}_{0.62} \mathrm{Ga}_{0.38} \mathrm{As}_{0.82} \mathrm{P}_{0.18}$ & $\begin{array}{l}1500 \\
1500\end{array}$ & $\begin{array}{l}2 \times 10^{18} / \mathrm{P} \\
1 \times 10^{18} / \mathrm{P}\end{array}$ \\
\hline \multirow[t]{2}{*}{ Absorption } & $\operatorname{In}_{0.53} \mathrm{Ga}_{0.47} \mathrm{As}$ & $\rightarrow \mathrm{In}_{0.53} \mathrm{Ga}_{0.47} \mathrm{As}^{1), *}$ & $\begin{array}{l}3000 \\
3000\end{array}$ & $\begin{array}{c}5 \times 10^{17} / \mathrm{P} \\
2.5 \times 10^{17} / \mathrm{P}\end{array}$ \\
\hline & & $\mathrm{In}_{0.53} \mathrm{Ga}_{0.47} \mathrm{As}$ & 1500 & $1 \times 10^{16} / \mathrm{N}$ \\
\hline Transition & & InGaAsP & 300 & $1 \times 10^{16} / \mathrm{N}$ \\
\hline Cliff & & InP & 500 & $1 \times 10^{17} / \mathrm{N}$ \\
\hline \multirow[t]{2}{*}{ Collector } & & InP & 9000 & $1 \times 10^{16} / \mathrm{N}$ \\
\hline & & $\mathrm{InP}$ & 1000 & $1 \times 10^{18} / \mathrm{N}$ \\
\hline \multirow[t]{3}{*}{ N-Contact } & & InP & 9000 & $1 \times 10^{19} / \mathrm{N}$ \\
\hline & & InGaAs & 200 & $1 \times 10^{19} / \mathrm{N}$ \\
\hline & & $\mathrm{InP}$ & 2000 & $1 \times 10^{19} / \mathrm{N}$ \\
\hline Substrate & Sen & ni-insulating InP & & \\
\hline
\end{tabular}

1) $\mathrm{In}_{0.62} \mathrm{Ga}_{0.38} \mathrm{As}_{0.82} \mathrm{P}_{0.18} \rightarrow \mathrm{In}_{0.53} \mathrm{Ga}_{0.47} \mathrm{As}$ means $x$ varies linearly from 0.38 to 0.47 in the undepleted absorption layer, and $y$ varies from 0.82 to 1 correspondingly.

* The association of composition fractions with the constituent elements of the material is $\operatorname{In}_{1-\mathrm{x}} \mathrm{Ga}_{\mathrm{x}} \mathrm{As}_{\mathrm{y}} \mathrm{P}_{1-\mathrm{y}}$.

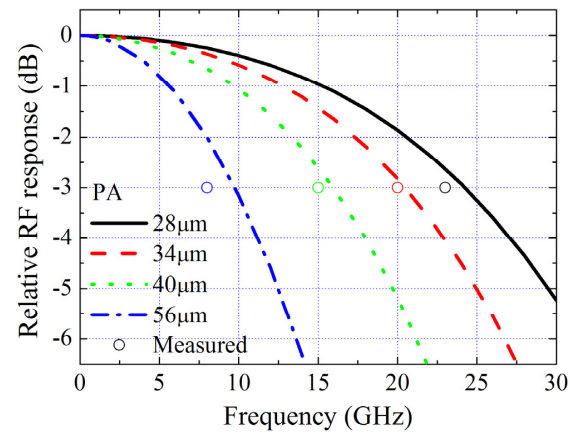

(a)

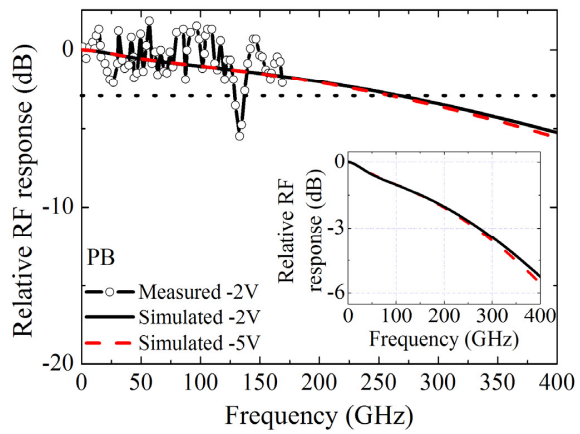

(b)

Fig. 1. Simulated modulation response of (a) PA with diameters of $28,34,40$, and $56 \mu \mathrm{m}$ at -6 $\mathrm{V}$ bias voltage and measured bandwidth [11], and (b) $\mathrm{PB}\left(16 \mu \mathrm{m}^{2}\right)$ at bias voltages of $-2 \mathrm{~V}$ and $-5 \mathrm{~V}$ and measured response [12] (y-axis keeps the same scale with Fig. 2b in [12], and the inset shows only the simulated results). The dip at $135 \mathrm{GHz}$ in the experimental curve is caused by the integrated coplanar waveguide [12]

On the other hand, the performance of high-output photodiodes may be impacted by high hole charge density due to high injection optical power. The effect of a high hole charge density in the p-type absorption would accelerate the electron diffusion and result in bandwidth enhancement under high power illumination [4]. Figure 2 shows the simulated modulation response at four illumination densities of 5000, 10000, 15000, and $20000 \mathrm{~W} / \mathrm{cm}^{2}$. It is clearly obvious that higher hole charge density indeed improves the bandwidth. Consequently, the physically-based simulations are reliable. It should be mentioned that although in the simulation, parasitic elements in actual circuits are not included, and the series resistance is used to fit the simulated bandwidth with measured data, the physical effects showing bandwidth changed with device active area, external bias voltage, and illumination 
which hole carriers that have slower transport velocity are removed and only electron carriers that have faster transport velocity are used. In UTC-PDs, $\tau_{\text {tr }}$ is usually dominated by the electron transport time in the absorption layer, because the electron drift time in the collector is negligible due to the overshoot effect. In the following, the graded structures design will be considered to improve the electron transport velocity in the absorption layer.

The graded structures include graded doping and graded bandgap. The graded doping can induce a built-in electric field, which can make electrons drift and move faster. For example, in Table 2, a large slope of linear graded doping profile is used in PB, which can create a built-in electric field from $\sim 2$ to $\sim 80 \mathrm{kV} / \mathrm{cm}$ [19]. Similarly, a step doping is adopted for PA as seen in Table 1. In contrast to the widely used graded doping, to the best of our knowledge, the absorption layer with graded-bandgap structure has not been adopted yet in actual UTCPDs. The potential gradient from the graded bandgap can also accelerate the electrons and further improve the device performance. For the InP/InGaAs material system, $\mathrm{In}_{1-\mathrm{x}} \mathrm{Ga}_{\mathrm{x}} \mathrm{As}_{\mathrm{y}} \mathrm{P}_{1-\mathrm{y}}$ or $\mathrm{In}_{1-\mathrm{x}-\mathrm{y}} \mathrm{Al}_{\mathrm{x}} \mathrm{Ga}_{\mathrm{y}} \mathrm{As}$ with lattice matched to InP can provide a range of bandgaps, which can be used to construct a continuous compositionally-graded bandgap structure. Here, the graded absorption layer using $\mathrm{In}_{1-\mathrm{x}} \mathrm{Ga}_{\mathrm{x}} \mathrm{As}_{\mathrm{y}} \mathrm{P}_{1-\mathrm{y}}$ will be considered.

The energy bandgap for the InP lattice matched $\operatorname{In}_{1-x} \mathrm{Ga}_{\mathrm{x}} \mathrm{As}_{\mathrm{y}} \mathrm{P}_{1-\mathrm{y}}$ is related to the composition fraction $x, y$ as follows [24],

$$
\begin{aligned}
E_{g}\left(\operatorname{In}_{1-x} \mathrm{Ga}_{x} \mathrm{As}_{y} \mathrm{P}_{1-y}\right) & =1.35+(0.642+0.758 x) x+(0.101 y-1.101) y \\
& -(0.28 x-0.109 y+0.159) x y .
\end{aligned}
$$

To design a graded $\mathrm{In}_{1-\mathrm{x}} \mathrm{Ga}_{\mathrm{x}} \mathrm{As}_{\mathrm{y}} \mathrm{P}_{1-\mathrm{y}}$ absorption layer, two basic conditions should be satisfied.

1) To maintain the lattice matched to InP, $x$ and $y$ should have the following relation [24],

$$
x=\frac{0.1896 y}{0.4176-0.0125 y} .
$$

2) To excite the interband transition by the light around $1.5-\mu \mathrm{m}$ wavelength, $E_{g}$ should be no more than $0.82 \mathrm{eV}$.

The above two conditions can be fulfilled within the range of $x$ from 0.38 to 0.47 , and correspondingly, the range of $y$ is from 0.82 to 1 . Therefore, the maximum potential difference is formed between $\operatorname{In}_{0.62} \mathrm{Ga}_{0.38} \mathrm{As}_{0.82} \mathrm{P}_{0.18}$ and $\operatorname{In}_{0.53} \mathrm{Ga}_{0.47} \mathrm{As}$.

To simulate the effect of graded bandgap, the linear compositionally-graded $\mathrm{In}_{0.62} \mathrm{Ga}_{0.38} \mathrm{As}_{0.82} \mathrm{P}_{0.18} \rightarrow \mathrm{In}_{0.53} \mathrm{Ga}_{0.47} \mathrm{As}$ is considered to substitute for the $\operatorname{In}_{0.53} \mathrm{Ga}_{0.47} \mathrm{As}$ absorption layer of PA $(0.9-\mu \mathrm{m}$ undepleted part) and PB. Note that the graded InAlGaAs transition layer is also replaced with InGaAsP in PB structure. For clarity, the layer parameters of PA and PB with the graded bandgap absorption layers are also listed in Tables 1 and 2, respectively. If the top of the P contact layer is set as the origin of the depth $(d)$, the relations between the composition fraction $x$ and $d$ (in $\mu \mathrm{m}$ ) are $2.762 x-0.276 d=1$, $d \in[0.18,1.08] \mu \mathrm{m}$ for PA, and $3.247 x-1.948 d=1, d \in[0.12,0.27] \mu \mathrm{m}$ for PB. The fraction $y$ is calculated according to condition 1, i.e. (2). The electric field distributions and the band diagram in the $0.15-\mu \mathrm{m}$-thickness absorption layer (from 0.12 to $0.27 \mu \mathrm{m}$ ) of PB are plotted in Fig. 3. It is seen that the built-in electric filed induced by the graded doping is greater than $\sim 2 \mathrm{kV} / \mathrm{cm}$ as mentioned above [19]. When the graded bandgap is introduced, the built-in electric field is increased to greater than $\sim 4 \mathrm{kV} / \mathrm{cm}$, which will lead to the improvement in bandwidth. It is also seen that the potential gradient in conduction band is enhanced, which means that the graded bandgap will mainly enhance the transport of electrons. 


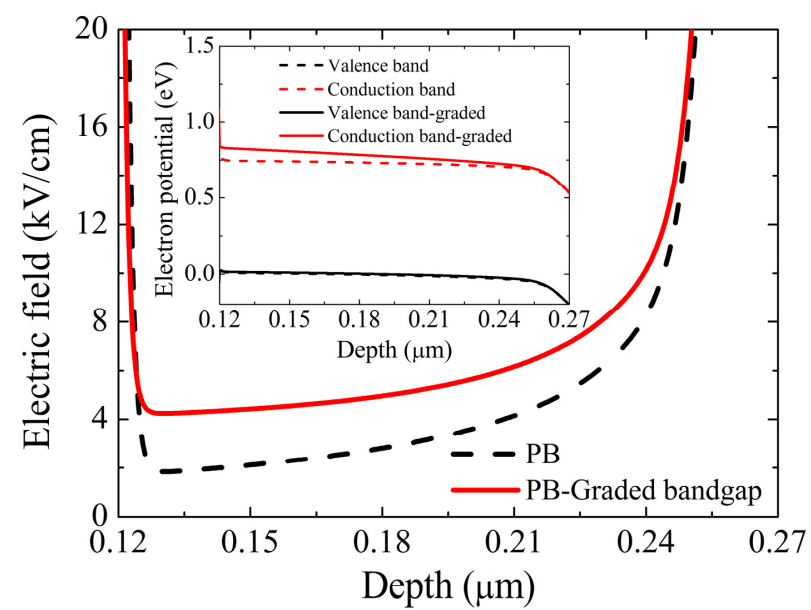

Fig. 3. Distribution of electric field in the absorption layer of PB with and without gradedbandgap design. Inset shows the band diagram of the absorption layer with and without graded bandgap design. External bias voltage: $-2 \mathrm{~V}$.

To show the performance improvement of using the graded bandgap design for PA and PB as shown in Tables 1 and 2, simulated modulation responses with comparison to PA and PB without using graded bandgap are shown in Fig. 4. It is seen that the bandwidths are increased from $24.3 \mathrm{GHz}$ to $26.1 \mathrm{GHz}$ and from $273 \mathrm{GHz}$ to $373 \mathrm{GHz}$ for PA and PB, respectively. The improvement of $\mathrm{PB}$ is $36.6 \%$, much more significant than PA for which the improvement is $7.4 \%$. This is attributed to a larger slope of the potential gradient in a thinner absorption layer in PB compared to PA.

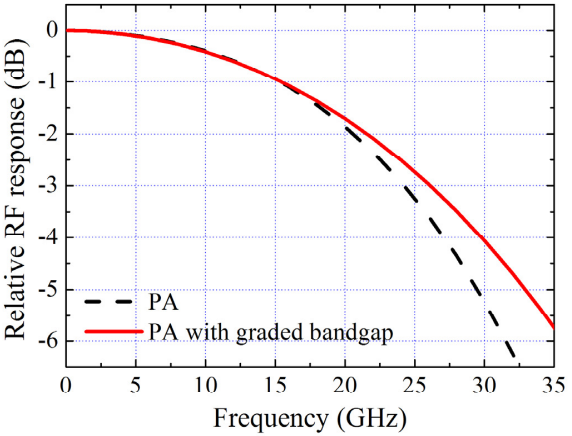

(a)

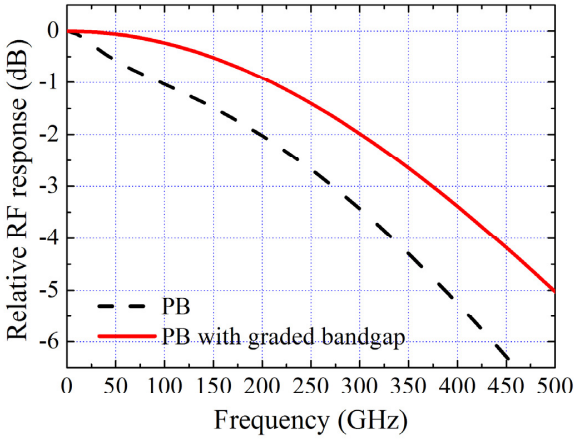

(b)

Fig. 4. Simulated modulation response for (a) PA (28- $\mu \mathrm{m}$ diameter) at $-6 \mathrm{~V}$ bias, (b) PB at $-2 \mathrm{~V}$ bias, using and without using graded-bandgap absorption layer.

\subsection{Modified graded bandgap design}

It is shown that the graded bandgap will contribute significantly to the built-in electric field if larger slope of the potential gradient is induced. The slope can be increased by using a larger potential difference or shortening the graded region. However, the maximum potential difference is determined by condition 2 , and shortening the graded region by simply reducing the thickness of the absorption layer will sacrifice the device responsivity. As shown in Fig. 3, the improvement of electric field after the first $0.12-\mu \mathrm{m}$ thickness (after the depth of $0.24 \mu \mathrm{m}$ ) of the absorption layer is not significant by using the graded bandgap. Thus, an efficient way to implement the gradient is to use the graded design (including graded bandgap and graded doping) only in the first $0.12-\mu \mathrm{m}$-thickness region and leave the rest $0.03-\mu \mathrm{m}$-thickness region ungraded. Similar design is also applied to PA in which the $0.9-\mu \mathrm{m}$-thickness 
undepleted absorption layer is split into an $0.82-\mu \mathrm{m}$-thickness graded region and an $0.08-\mu \mathrm{m}$ thickness ungraded region. The effect on the electric field distribution, in the absorption layer, by using this modified graded bandgap (graded doping is also modified, similarly hereinafter) for PB is shown in Fig. 5. It is seen that the modified graded bandgap results in the increase of the build-in electric field, and also the increase of the electric field is the largest at around depth of $0.24 \mu \mathrm{m}$ that is corresponding to the graded absorption layer. Using the modified graded bandgap, the improvements of bandwidth for both the devices can be found in Fig. 6, in which modulation responses of PA and PB are shown with comparison to the graded bandgap. It is seen that this modification of the graded absorption layers leads to additional 1$\mathrm{GHz}(4.1 \%)$ and 26-GHz (9.5\%) bandwidth improvements for PA and PB, respectively.

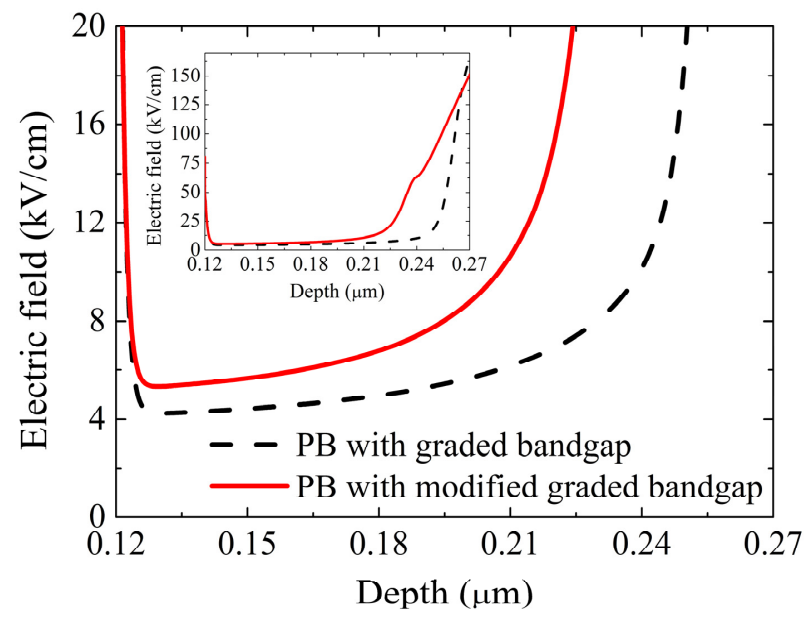

Fig. 5. Distribution of electric field in absorption layer of PB with graded and modified graded bandgap. Inset is a large-scale view in y axis.

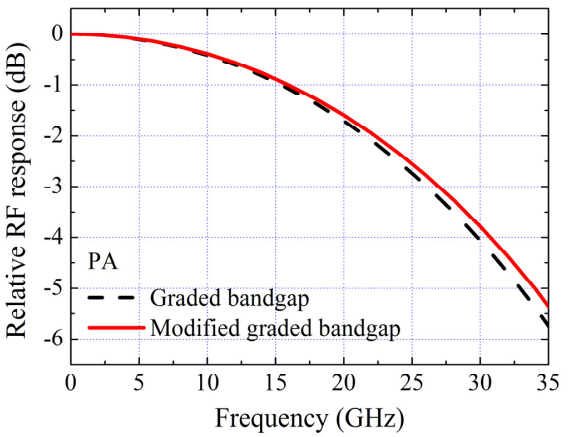

(a)

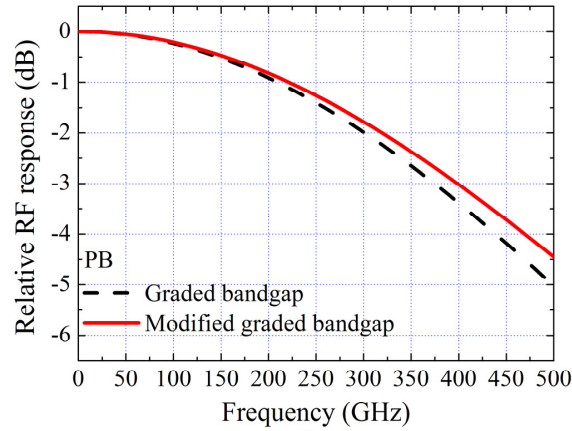

(b)

Fig. 6. Simulated modulation response of (a) PA (28- $\mu \mathrm{m}$ diameter) at $-6 \mathrm{~V}$ bias voltage (b) PB at $-2 \mathrm{~V}$ bias voltage using modified graded bandgap with comparison to graded bandgap.

It is shown above that graded bandgap leads to enhancement of modulation response, and particularly much more significant for thin absorption layer. On the other hand, the modulation response may be limited by RC delay, mainly induced by the collector layer, thus use of graded doping and/or graded bandgap that enhance the electron diffusion may not improve the modulation performance. As an example, we consider PB in [12] in which graded doping was used, where it was found that the modulation response might be limited by RC delay. Compared PB with similar devices in [19], much lower contact resistance and parasitic capacitance are achieved. Taking those values in the equivalent circuit in [12], a RC bandwidth of around $340 \mathrm{GHz}$ is achieved, much higher than the measured $250 \mathrm{GHz}$. Also, if 
sacrificing a small part of the output power by reducing the device active area from $16 \mu \mathrm{m}^{2}$ to $14 \mu \mathrm{m}^{2}$, the RC bandwidth can reach $380 \mathrm{GHz}$. Therefore, it is highly possible that improving the electron transit speed in the absorption layer by using graded bandgap is still an effective way to increase the bandwidth for many designs of broadband photodiodes.

\subsection{Saturation current}

With increased electron speed, the saturation current is also improved. The saturation current performance of $\mathrm{PB}$ is simulated indirectly by solving the photocurrent under DC illumination. Since the simulator simply takes the imaginary part $(\kappa)$ of the refractive index to evaluate the optic-to-electric (O-E) conversion efficiency, in the simulations $\kappa$ is determined by fitting the responsivity (the slopes of the curves in Fig. 7) to the measured result in [12]. $\kappa$ is assumed the same for $\mathrm{In}_{0.53} \mathrm{Ga}_{0.47} \mathrm{As}$ and InGaAsP. Both InGaAsP (low P fraction for our structure) and $\mathrm{In}_{0.53} \mathrm{Ga}_{0.47} \mathrm{As}$ are direct bandgap materials and have similar band structures, therefore, this approximation is acceptable and may not lead to significant error in O-E conversion efficiency of InGaAsP. As shown in Fig. 7, the DC current increases linearly with the increasing light power till the inflection point, and then the current becomes to saturate. The reduction of the slope indicates a decreased responsivity due to the space charge effect from the carrier storage at high-optical input [4]. Such effect can also lead to the degradation of the bandwidth. The DC saturation current for PB is $35 \mathrm{~mA}$. By using the graded bandgap structure, the DC saturation current can be improved to $49 \mathrm{~mA}$.

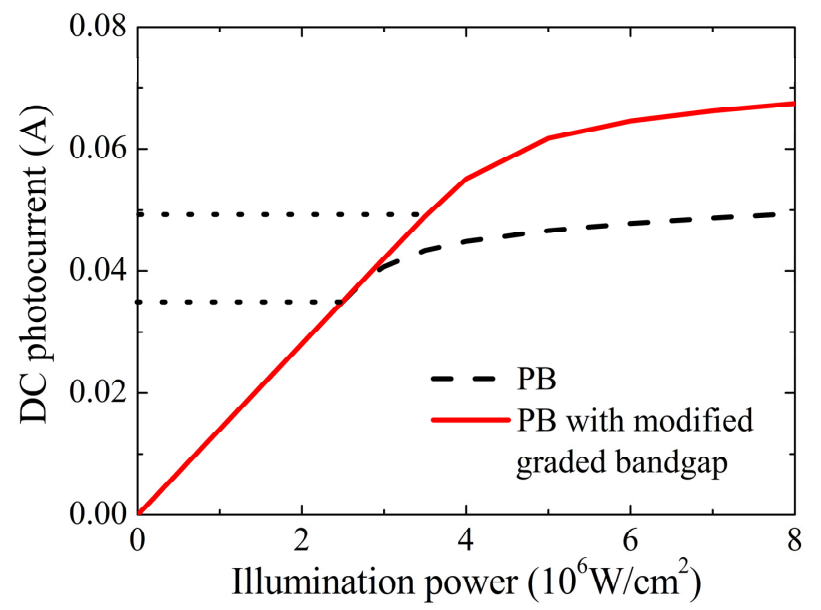

Fig. 7. Under DC illumination, simulated photocurrent versus illumination power of PB.

The improvement in the saturation current by the graded bandgap design may be understood as follows. It is known that the saturation effect is due to the space charge in the depleted collector. High electron concentration caused by high power illumination will reduce the electric field in the collector, and thus leading to the saturation. Basically, there are critical values for electric field $E_{c}$ and for electron concentration $n_{c}$. When the electron concentration in the collector increases, the field in the collector reduces and finally falls below $E_{c}$, where the saturation occurs. $E_{c}$ is usually determined by the material which is InP here. However, $n_{c}$ may vary as external conditions. For example, higher bias voltage usually leads to higher saturation current. This means $n_{c}$ is a function of bias voltage. This could be used to explain our situation. Under moderate illumination power, the external bias voltage mainly falls on the depleted collector. But when the illumination power increases, the bias voltage may be shared 


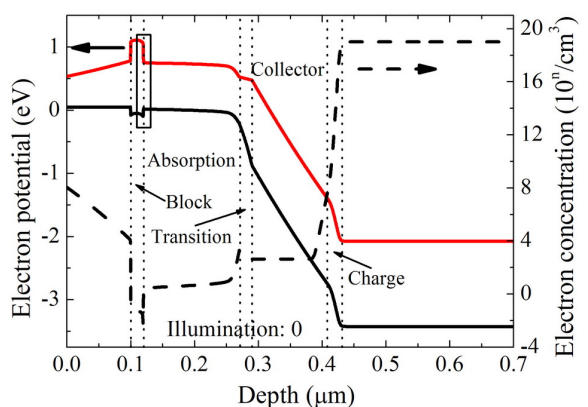

(a)

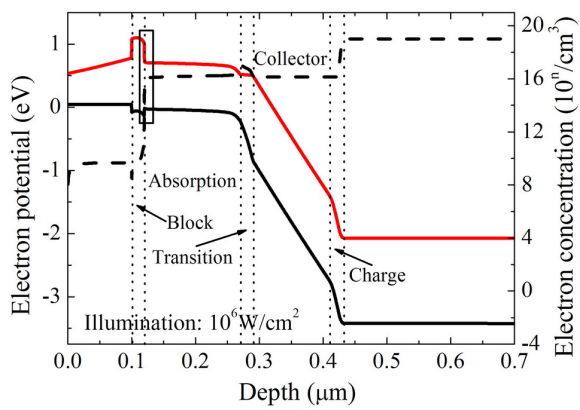

(c)

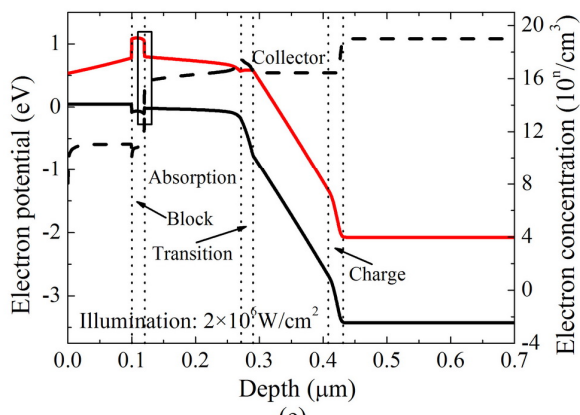

(e)

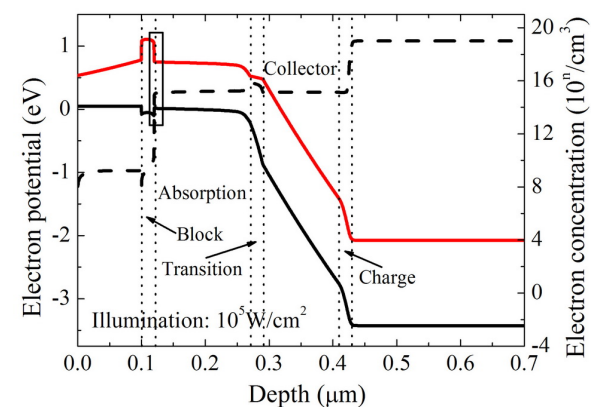

(b)

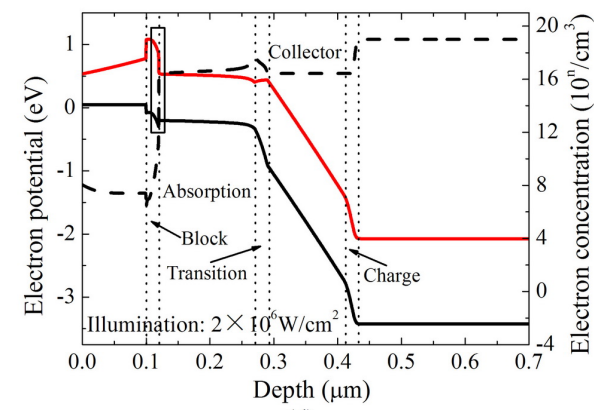

(d)

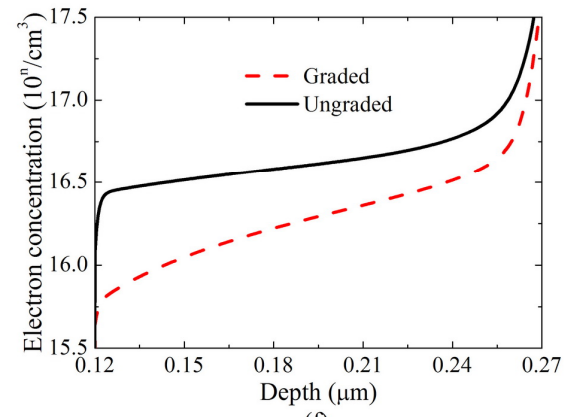

(f)

Fig. 8. Band diagrams (solid) and electron distributions (dashed) under different illumination levels for ungraded and graded bandgap structures. The bias voltage is $-2 \mathrm{~V}$. (a) Ungraded, no illumination, (b) Ungraded, illumination power: $10^{5} \mathrm{~W} / \mathrm{cm}^{2}$ (c) Ungraded, illumination power: $10^{6} \mathrm{~W} / \mathrm{cm}^{2}$ (d) Ungraded, illumination power: $2 \times 10^{6} \mathrm{~W} / \mathrm{cm}^{2}$ (e) Graded, illumination power: $2 \times 10^{6} \mathrm{~W} / \mathrm{cm}^{2}$ (f) Comparison of electron concentrations, illumination power: $2 \times 10^{6} \mathrm{~W} / \mathrm{cm}^{2}$.

The position of each layer is marked in the figures.

by the junction at the interface of the block layer and the absorption layer, which is explained below. For the structure without graded bandgap biased at $-2 \mathrm{~V}$ voltage, as shown in Figs. 8(a)-8(d), when the illumination is increased, the concentration of photo-generated carriers in the absorption layer increases. For the case of no illumination, it is seen that the collector (as well as the transition layer which is actually a part of the effective collector) is depleted, and the electron concentration is around $10^{3} / \mathrm{cm}^{3}$. For the case of illumination power density of $10^{5} \mathrm{~W} / \mathrm{cm}^{2}$, the photo generation occurs. The electron concentrations in the absorption layer and the collector increase, and the device operates. For the case of illumination power density of $10^{6} \mathrm{~W} / \mathrm{cm}^{2}$, the device operates normally. But there is a slight change at the interface between the block layer and the absorption layer, which is indicated by the boxes in the figures. The electron concentration in the absorption layer goes higher as the illumination power increases, thus electron diffusion into the block layer occurs, which forms a junction at the interface. For the case of illumination power density of $2 \times 10^{6} \mathrm{~W} / \mathrm{cm}^{2}$, as the illumination 
power increases continuously, the junction becomes significant because of the rise of the electron concentration. This junction suffers partial of the external bias, which lowers the electric field in the collector. As seen clearly in Fig. 8(d), the electric field at the p side of the collector (in the transition layer) is reduced compared to that in Fig. 8(a). Although at this illumination level, the electric field in the collector is not affected much and the output current is still not saturated, further increasing the illumination power will make this junction more depleted and experience more external bias. This effect will reduce the electric field in the collector, pushing the current saturation appearing early. If considering the graded bandgap design, the situation discussed above could be relaxed. For the graded bandgap structure, the electron concentration also increases with increasing illumination power. However, the shortened electron transition time by the potential gradient will reduce the electron concentration in the absorption layer greatly, which is shown in Figs. 8(e) and 8(f). At the same illumination level $\left(2 \times 10^{6} \mathrm{~W} / \mathrm{cm}^{2}\right)$, the electron concentration is reduced by more than 3 times. This effect is especially significant in the vicinity of the interface between the block layer and the absorption layer. Therefore, the electron diffusion into the block layer is reduced accordingly, and the junction is also suppressed. That is to say, at the same illumination power, more external bias voltage falls on the collector for graded structure than ungraded structure, and the electric field in the collector of graded structure is maintained correspondingly. This may be the possible reason to explain the improvement of saturation current by the graded bandgap design.

Although the DC saturation current is simulated instead of the AC saturation current, both of them are closely affected by the space-charge effect. Thus, the reduction of the responsivity is an effective indicator of AC saturation current [11]. The actual improvement of the saturation current might be much lower due to several factors such as voltage swing and thermal effect as stated in [11]. To realize high-output power performance, the cliff layer design in [11] may be employed to further increase the saturation current. However, in practical use, the saturation current for such small device is usually limited by thermal failure. Therefore, including the heat dissipation problem in the simulation is necessary to evaluate the saturation current accurately. Our simulated results without considering the heat could be useful providing new packaging techniques with efficient heat sinking are developed.

\subsection{Fabrication considerations}

It should be noted that both $\operatorname{In}_{1-x} \mathrm{Ga}_{\mathrm{x}} \mathrm{As}_{\mathrm{y}} \mathrm{P}_{1-\mathrm{y}}$ and $\operatorname{In}_{1-\mathrm{x}-\mathrm{y}} \mathrm{Al}_{\mathrm{x}} \mathrm{Ga}_{\mathrm{y}} \mathrm{As}$ can be grown by epitaxy techniques, such as MBE, MOVPE, or MOCVD, etc. For $\mathrm{In}_{1-\mathrm{x}} \mathrm{Ga}_{\mathrm{x}} \mathrm{As}_{\mathrm{y}} \mathrm{P}_{1-\mathrm{y}}$ grown by MOCVD, the quality of linearly grading can be ensured except for large composition ranges, which can meet the requirement of the graded absorption layer. While for the thin transition layer with a large grading range from $\mathrm{In}_{0.53} \mathrm{Ga}_{0.47} \mathrm{As}$ to $\mathrm{InP}$, an alternative method is using step grading. The differences in simulated performances between the linearly continuous grading and a 3step grading in transition layer are negligible. In addition, $\operatorname{In}_{1-x-y} \mathrm{Al}_{\mathrm{x}} \mathrm{Ga}_{\mathrm{y}} \mathrm{As}$ is easier to control in MBE. Therefore, the use of $\operatorname{In}_{1-x-y} \mathrm{Al}_{x} \mathrm{Ga}_{\mathrm{y}} \mathrm{As}$ graded layer may be more practical and economical. The design method of a continuous compositionally-graded $\operatorname{In}_{1-x-y} \mathrm{Al}_{\mathrm{x}} \mathrm{Ga}_{\mathrm{y}} \mathrm{As}$ absorption layer is similar to that of $\operatorname{In}_{1-\mathrm{x}} \mathrm{Ga}_{\mathrm{x}} \mathrm{As}_{\mathrm{y}} \mathrm{P}_{1-\mathrm{y}}$.

\section{Conclusion}

Design of broadband and high-output power UTC-PDs is of crucial importance to future high-speed communication systems. Different from equivalent circuit models requiring fitted values from measurements, it is shown that physically-based modeling of UTC-PDs can predict the device performance as long as the device structural parameters are known, and the simulated results can be directly used in the device design. Using the physical modeling, the graded bandgap absorption layer has been considered and investigated, and it is shown that such design is especially effective for devices with thin absorption layers. The photodiode performance can be further improved by using the modified graded structure. The total improvement of $46.1 \%$ in bandwidth, by using the proposed modified graded bandgap absorption layer for $\mathrm{PB}$, is predicted. With the reduced electron transit time, the saturation 
current is also improved by $40 \%$. Using the PB structure in [12] with our proposed modified graded bandgap, 399-GHz bandwidth and 49-mA DC saturation current are predicted by the physically-based modeling. Our future work is to fabricate photodiodes with modified graded bandgap structure and further verify our proposed design.

\section{Acknowledgment}

This work was supported in part by a team research project of Quebec Fonds de recherche nature et technologies (FQRNT).

\#184193 - \$15.00 USD Received 28 Jan 2013; revised 4 Mar 2013; accepted 5 Mar 2013; published 13 Mar 2013

(C) 2013 OSA

25 March 2013 / Vol. 21, No. 6 / OPTICS EXPRESS 6954 